\title{
Modified Bloch equations in presence of a nonstationary bath
}

\author{
Jyotipratim Ray Chaudhuri, Suman Kumar Banik, Bimalendu Deb* and Deb Shankar Ray ${ }^{\dagger}$ \\ Indian Association for the Cultivation of Science, Jadavpur, Calcutta 700032, INDIA.
}

\begin{abstract}
Based on the system-reservoir description we propose a simple solvable microscopic model for a nonequilibrium bath. This captures the essential features of a nonstationary quantum Markov process. We establish an appropriate generalization of the fluctuation-dissipation relation pertaining to this process and explore the essential modifications of the Bloch equations to reveal the nonexponential decay of the Bloch vector components and transient spectral broadening in resonance fluorescence. We discuss a simple experimental scheme to verify the theoretical results.
\end{abstract}

PACS number(s) : 32.80.-t,42.65.An

* present address : Department of Chemical Physics, Weizmann Institute of Science, Rehovot, Israel.

${ }^{\dagger} \mathrm{e}-\mathrm{mail}:$ pcdsr@mahendra.iacs.res.in 


\section{INTRODUCTION}

The dynamics of most of the quantum optical phenomena are based on two fundamental processes; the coherent interaction between the system (atom/molecule) and the field mode (classical/quantum) and the incoherent dissipation of the system. The latter is traditionally modeled in terms of the wellknown system-reservoir theory within the appropriate finite temperature quantum statistical scheme [1] [5]. Besides thermal reservoirs, the non-thermal reservoirs [4.5] have also been found to be important in connection with the development of correlated emission lasers [4] and squeezed light fields [5]. The essential underlying assumption about the bath, be it thermal or nonthermal, is that it is considered to be in a state of equilibrium throughout the process. Very recently a solvable microscopic model for a nonequilibrium bath has been proposed [6] to explore classically, the influence of an initial nonequilibrium excitation in a complex system on the relaxation of a specific quantity of interest. In the present paper we extend this treatment to a quantum optical context. Since the initial excitation creates a nonequilibrium energy density fluctuation distribution which imparts nonstationarity of the bath, it is expected that optical Bloch equations which take into account of both the coherent interaction and the relaxation processes within a simplified description of a twolevel scheme, are likely to be modified by the nonstationarity of the bath [6,7]. Based on a quantum version of the model we study this essential modification of the optical Bloch equations and explore some of the relevant consequences.

We thus consider a two-level system in contact with a bath which is not in a thermal Boltzmann distribution. This nonequilibrium bath is effectively realized in terms of a semi-infinite dimensional broad-band reservoir which is subsequently kept in contact with a standard thermal bath which allows the nonthermal bath to relax with a characteristic time scale. The important separation of the time scales of fluctuations of the nonequilibrium and the thermal bath is that [6] the former remains effectively stationary on the fast correlation of the thermal noise. The model captures the essential 
features of a nonstationary quantum Markov process. The physical situation that has been addressed is the following. At $t=0$ the excitation is switched on and the intermediate bath is thrown into a nonstationary state. We then follow the coherent dynamics of a classical laser-driven near-resonant two-level system interrupted by incoherent emissive processes due to nonequilibrium intermediate modes after $t>0$ to observe the influence of relaxation of these modes on the transient characteristics of the system. We show that the decay of the Bloch vector components is nonexponential in character so long as the nonstationarity persists. In addition, the nonstationarity of the bath results in timedependence of the diffusion coefficient which manifests itself in the transient resonance fluorescence spectra of the two-level system. The underlying physical mechanism of the transient characteristics can be understood with the help of a generalized nonequilibrium fluctuation-dissipation relation pertaining to this nonstationary quantum Markov process. In the long time limit one, however, recovers the standard Bloch equations and the spectral features.

The outline of the paper is as follows : In Sec.II we discuss the model for nonequilibrium bath and the generalization of the fluctuation-dissipation relation corresponding to the nonstationary process. The application of the model to explore the modification of the optical Bloch equations and the transient spectral characteristics of resonance fluorescence have been carried out in Sec.III. In Sec.IV we propose a simple experimental scheme to verify the theoretical results on nonexponential decay and transient broadening effect. The paper is concluded with a summary of the main results.

\section{RELAXATION OF A TWO-LEVEL ATOM IN PRESENCE OF A NONSTATIONARY BATH}

To start with we consider a model two-level atom (the system) coupled to a set of relaxing modes considered as a semi-infinite dimensional system which effectively constitutes a nonequilibrium 
reservoir. This, in turn, is in contact with a thermally equilibrated reservoir. Both the reservoirs are composed of two sets of harmonic oscillators characterized by the frequency sets $\left\{\omega_{j}\right\}$ and $\left\{\Omega_{j}\right\}$ for the equilibrium and nonequilibrium bath, respectively. The total Hamiltonian is given by

$$
\begin{aligned}
H_{0} & =\frac{1}{2} \hbar \omega_{0} \sigma_{z}+\hbar \sum_{j} \omega_{j} b_{j}^{\dagger} b_{j}+\hbar \sum_{\mu} \Omega_{\mu} c_{\mu}^{\dagger} c_{\mu} \\
& +\hbar \sum_{\mu} g_{\mu}\left(\sigma_{+} c_{\mu}+\sigma_{-} c_{\mu}^{\dagger}\right)+\hbar \sum_{j} \sum_{\mu} \alpha_{j \mu}\left(b_{j}^{\dagger} c_{\mu}+b_{j} c_{\mu}^{\dagger}\right) .
\end{aligned}
$$

The Hamiltonian is essentially a simpler quantum version of the model used in [6] with two-level atom as the system. The first term on the right-hand side describes the system mode with characteristic frequency $\omega_{0}$. The second and the third terms represent the thermal and the nonequilibrium linear modes. The next two terms represent the coupling of the nonequilibrium bath with the system mode and the thermal bath where the coupling constants are $g_{\mu}$ and $\alpha_{j \mu}$, respectively. In writing down the Hamiltonian we have made use of the rotating wave approximation.

The Heisenberg equations of motion for the system and the reservoir operators are given by

$$
\begin{aligned}
\dot{\sigma}_{+}(t) & =i \omega_{0} \sigma_{+}-i \sum_{\mu} g_{\mu} c_{\mu}^{\dagger} \sigma_{z} \\
\dot{\sigma}_{-}(t) & =-i \omega_{0} \sigma_{-}+i \sum_{\mu} g_{\mu} c_{\mu} \sigma_{z} \\
\dot{\sigma}_{z}(t) & =-2 i \sum_{\mu} g_{\mu} c_{\mu} \sigma_{+}+2 i \sum_{\mu} g_{\mu} c_{\mu}^{\dagger} \sigma_{-}, \\
\dot{b}_{j}(t) & =-i \omega_{j} b_{j}-i \sum_{\mu} \alpha_{j \mu} c_{\mu} \\
\dot{c}_{\mu}(t) & =-i \Omega_{\mu} c_{\mu}-i g_{\mu} \sigma_{-}-i \sum_{j} \alpha_{j \mu} b_{j} .
\end{aligned}
$$

Making use of the formal integral of Eq.(5) for $b_{j}(t)$ in Eq.(6) we obtain

$$
\begin{aligned}
\dot{c}_{\mu}(t)= & -i \Omega_{\mu} c_{\mu}-i g_{\mu} \sigma_{-}-i \sum_{j} \alpha_{j \mu} e^{-i \omega_{j}\left(t-t_{0}\right)} b_{j}\left(t_{0}\right) \\
& -\sum_{j} \sum_{\nu} \alpha_{j \mu} \alpha_{j \nu} \int_{t_{0}}^{t} d t^{\prime} c_{\nu}\left(t^{\prime}\right) e^{-i \omega_{j}\left(t-t^{\prime}\right)} .
\end{aligned}
$$


Taking into consideration that the interference time of $\sum_{j} \alpha_{j \mu} \alpha_{j \nu} e^{-i \omega_{j}\left(t-t^{\prime}\right)}$ is much smaller than the time over which the significant phase and amplitude modulation of the linear modes $c_{\mu}(t)$ takes place, the last term in Eq.(7) can be identified as a relaxation term in the usual way [1] with the damping constant

$$
\gamma_{\mu \nu}^{c}=\pi \alpha_{\nu \mu}\left(\Omega_{\nu}\right) \alpha_{\nu \nu}\left(\Omega_{\nu}\right) D\left(\Omega_{\nu}\right)
$$

where $D\left(\Omega_{\nu}\right)$ represents the density of states of the equilibrium modes evaluated at $\Omega_{\nu}$. Thus one can write down the Langevin equation for the relaxing mode $c_{\mu}$ as follows;

$$
\dot{c}_{\mu}(t)=-i \Omega_{\mu} c_{\mu}(t)-i g_{\mu} \sigma_{-}(t)-\sum_{\nu} \gamma_{\mu \nu}^{c} c_{\nu}(t)+f_{\mu}(t)
$$

Here the last term $f_{\mu}(t)$ represents the usual noise operator arising out of the coupling of the relaxing modes with the thermal bath modes as given by

$$
f_{\mu}(t)=-i \sum_{j} \alpha_{j \mu} e^{-i \omega_{j}\left(t-t_{0}\right)} b_{j}\left(t_{0}\right)
$$

where the reservoir average of $f_{\mu}(t)$ is zero, i.e.,

$$
\left\langle f_{\mu}(t)\right\rangle_{B}=0
$$

Here by average $\langle O(t)\rangle_{B}$ of an operator $O(t)$ we mean

$$
\langle O(t)\rangle_{B}=\operatorname{Tr}\left\{O(t) \rho_{B}\right\} \text {, where } \rho_{B}=\Pi_{j} \exp \left\{\left(-\hbar \omega_{j} b_{j}^{\dagger} b_{j}\right) / K T\right\} /\left[1-\exp \left(-\frac{\hbar \omega_{j}}{K T}\right)\right]
$$

$\rho_{B}$ is the thermal operator for initial density matrix for the thermal bath ( initial density for the thermal bath $\left\{b_{j}\right\}$, nonequilibrium bath $\left\{c_{\mu}\right\}$ and the system are assumed to be factorizable ).

We now make the following approximations. The cross-terms which involve rapidly evolving imaginary exponentials in the summation among the bath modes in Eq.(9) are neglected with respect to the diagonals, slowly evolving terms. This secular approximation is the usual one made in the 
context of master equations for baths [1]. It is wellknown [1] that this approximation is valid in the limit of weak coupling $\left(\left|\alpha_{\mu \nu}\right| \ll\left|\Omega_{\nu}\right|\right)$ and of a 'flat' bath spectrum for which $\left|\alpha_{\mu \nu}\right| \approx\left|\alpha_{\mu^{\prime} \nu^{\prime}}\right|$.

Taking into consideration of the above approximations the Langevin equation for the relaxing modes, Eq.(9), can be written in the following form,

$$
\dot{c}_{\mu}(t)=-i \Omega_{\mu} c_{\mu}(t)-i g_{\mu} \sigma_{-}(t)-\gamma_{\mu \mu}^{c} c_{\mu}(t)+f_{\mu}(t)
$$

To explore the influence of an initial excitation of the intermediate reservoir and its relaxation, we now consider the evolution of these linear modes $c_{\mu}$ in terms of Eq.(12), which allows a formal solution of the following form [6]

$$
c_{\mu}(t)=c_{\mu}^{s}(t)+c_{\mu}\left(t_{0}\right) e^{\left(-i \Omega_{\mu}-\gamma_{\mu \mu}^{c}\right)\left(t-t_{0}\right)}-i g_{\mu} \int_{t_{0}}^{t} d t^{\prime} e^{\left(-i \Omega_{\mu}-\gamma_{\mu \mu}^{c}\right)\left(t-t^{\prime}\right)} \sigma_{-}\left(t^{\prime}\right)
$$

The first term on the right hand side in the absence of the coupling of the system mode represents the ( long time ) stationary stochastic solution of the form

$$
c_{\mu}^{s}(t)=c_{\mu}^{s} e^{-i\left[\Omega_{\mu}\left(t-t_{0}\right)+\phi_{\mu}^{s}\right]},
$$

where the amplitude $c_{\mu}^{s}$ (operator) and the phases $\phi_{\mu}^{s}$ (c-number) are assumed to be randomly distributed [6]. The random distribution of phases and amplitudes in the stationary regime makes Eq.(13) an instantaneous solution. The second term on the right hand side in Eq.(13) carries the information of relaxation of the $c_{\mu}$ modes due to their coupling to the thermal bath and is in the form of a typical "memory-type term" [see the discussion later]. The latter is not to be confused with the usual memory term ( or kernel ) commonly arising out of the frequency dependence of friction. The third term on the other hand represents the effect of coupling of the system mode to the nonequilibrium reservoir.

We now substitute this solution (13) in Eq.(2) to obtain the equation of motion for the system operator in the usual way as 


$$
\dot{\sigma}_{+}(t)=i \omega_{0} \sigma_{+}(t)-\Gamma \sigma_{+}(t)+Z^{\dagger} \sigma_{z}(t)
$$

with

$$
\Gamma=\pi g^{2}\left(\omega_{0}\right) \rho\left(\omega_{0}\right)
$$

where the $\rho(\Omega)$ represents the density of relaxing intermediate oscillator modes. We assume further the weak dependence of $\gamma_{\mu \mu}^{c}$ on the modes to perform the integration over $\Omega$.

$\Gamma$ can be identified as a dissipation constant of the system mode due to the fluctuation of these modes. Also note that

$$
Z^{\dagger}(t)=-i \sum_{\mu} g_{\mu}\left[c_{\mu}^{s}(t)+c_{\mu}^{\dagger}\left(t_{0}\right) e^{\left(i \Omega_{\mu}-\gamma_{\mu \mu}^{c}\right)\left(t-t_{0}\right)}\right]
$$

is the noise operator for the nonequlibrium bath modes with $\left\langle Z^{\dagger}(t)\right\rangle_{N R}=0$. Here by $\langle O(t)\rangle_{N R}$ we mean

$$
\langle O(t)\rangle_{N R}=\operatorname{Tr}\left\{O(t) \rho_{c}\right\}, \text { where } \rho_{c}=\Pi_{\mu} \exp \left\{\left(-\hbar \Omega_{\mu} c_{\mu}^{\dagger} c_{\mu}\right) / K T\right\} /\left[1-\exp \left(-\frac{\hbar \Omega_{\mu}}{K T}\right)\right]
$$

Here $\rho_{c}$ is the initial thermal density operator for the nonequilibrium bath.

We proceed similarly to obtain the other equations of motion for system operators, $\sigma_{-}$and $\sigma_{+}$as,

$$
\begin{aligned}
& \dot{\sigma}_{-}(t)=-i \omega_{0} \sigma_{-}(t)-\Gamma \sigma_{-}(t)+Z(t) \sigma_{z}, \\
& \dot{\sigma}_{z}(t)=-2 \Gamma\left(1+\sigma_{z}\right)+2 Z(t) \sigma_{+}(t)+2 Z^{\dagger}(t) \sigma_{-}(t) .
\end{aligned}
$$

Introducing the slowly varying operators as

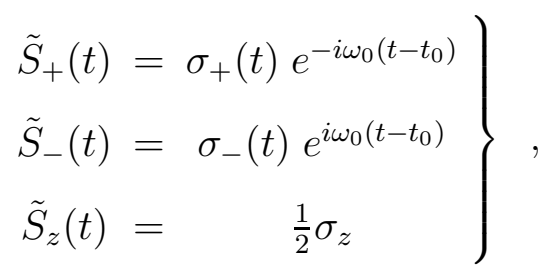


we obtain the following Langevin equations,

$$
\left.\begin{array}{ll}
\dot{\tilde{S}}_{+}(t)= & -\Gamma \tilde{S}_{+}(t)+2 \tilde{\xi}^{\dagger}(t) \tilde{S}_{z}(t) \\
\dot{\tilde{S}}_{-}(t)= & -\Gamma \tilde{S}_{-}(t)+2 \tilde{\xi}(t) \tilde{S}_{z}(t) \\
\dot{\tilde{S}}_{z}(t)= & -2 \Gamma\left[\frac{1}{2}+\tilde{S}_{z}(t)\right]+\tilde{\xi}^{\dagger}(t) \tilde{S}_{-}(t)+\tilde{\xi}(t) \tilde{S}_{+}(t)
\end{array}\right\},
$$

where

$$
\tilde{\xi}^{\dagger}(t)=-i \sum_{\mu} g_{\mu}\left[c_{\mu}^{s \dagger}(t) e^{-i \omega_{0}\left(t-t_{0}\right)}+c_{\mu}^{\dagger}\left(t_{0}\right) e^{i\left(\Omega_{\mu}-\omega_{0}\right)\left(t-t_{0}\right)} e^{-\gamma_{\mu \mu}^{c}\left(t-t_{0}\right)}\right]
$$

The nonequilibrium generalization of the fluctuation-dissipation relation is now immediately apparent. Using Eq.(21) we have

$$
\begin{aligned}
\left\langle\tilde{\xi}^{\dagger}(t) \tilde{\xi}\left(t^{\prime}\right)\right\rangle_{N R}= & \sum_{\mu} g_{\mu}^{2}\left[\left\langle c_{\mu}^{s \dagger} c_{\mu}^{s}\right\rangle_{N R} e^{i\left(\Omega_{\mu}-\omega_{0}\right)\left(t-t^{\prime}\right)}\right. \\
& \left.+\left\langle c_{\mu}^{\dagger}\left(t_{0}\right) c_{\mu}\left(t_{0}\right)\right\rangle_{N R} e^{i\left(\Omega_{\mu}-\omega_{0}\right)\left(t-t^{\prime}\right)} e^{2 \gamma_{\mu \mu}^{c} t_{0}} e^{-\gamma_{\mu \mu}^{c}\left(t+t^{\prime}\right)}\right] .
\end{aligned}
$$

We denote the average photon number of the nonequilibrium bath by

$$
\bar{n}\left(\Omega_{\mu}, t_{0}\right)=\left\langle c_{\mu}^{\dagger}\left(t_{0}\right) c_{\mu}\left(t_{0}\right)\right\rangle_{N R}
$$

where $t_{0}$ signifies the dependence of average photon number of the nonequilibrium bath on its initial state of preparation. Also the steady state average photon number is given by

$$
\bar{n}\left(\Omega_{\mu}\right)=\left\langle c_{\mu}^{s \dagger} c_{\mu}^{s}\right\rangle_{N R}
$$

After replacing the summation by integration and $\gamma_{\mu \mu}^{c}$ by an average $\gamma$ in Eq.(22) we obtain in the usual way

$$
\left\langle\tilde{\xi}^{\dagger}(t) \tilde{\xi}\left(t^{\prime}\right)\right\rangle_{N R}=\left[\Gamma \bar{n}\left(\omega_{0}\right)+e^{-2 \gamma\left(t-t_{0}\right)} \Gamma \bar{n}\left(\omega_{0}, t_{0}\right)\right] \delta\left(t-t^{\prime}\right)
$$

Eq.(24) and $\langle\tilde{\xi}(t)\rangle=0$ summarizes the essential properties of the stochastic processes due to intermediate oscillator bath modes $\left\{c_{\mu}\right\}$. It is important to emphasize that the exponential term in 
Eq.(24) $\left[\exp \left\{-2 \gamma\left(t-t_{0}\right)\right\}\right]$ does not contain time-difference of the two different instants $t$ and $t^{\prime}$ over which the stochastic process is correlated. Thus this exponential term is not to be confused with $\exp \left\{-2 \gamma\left(t-t^{\prime}\right)\right\}$ which normally appears as a typical memory term in correlation function as

$$
\left\langle\xi(t) \xi\left(t^{\prime}\right)\right\rangle=\text { Constant } \times \exp \left[-2 \gamma\left(t-t^{\prime}\right)\right]
$$

in a non-Markovian stochastic process. The time-difference of the two instants $t$ and $t^{\prime}$ in the present study appear as an argument of a delta function $\left[\delta\left(t-t^{\prime}\right)\right]$ in Eq.(24) due to the use of standard broadband reservoirs. $t$ in the exponential function in Eq.(24) is rather a slow time variable, which makes the stochastic process due to $\left\{c_{\mu}\right\}$-modes nonstationary. In other words the correlation function in Eq.(24) is not invariant under time translation. We are thus concerned here with a nonstationary quantum Markov process. This consideration is essential for the application of Onsager's regression hypothesis for calculation of spectra with two-time correlation function in the present case as shown in the next section.

Rewriting $\Gamma \bar{n}\left(\omega_{0}, t_{o}\right)$ in Eq.(24) in terms of a deviation from its steady state value $\Gamma \bar{n}\left(\omega_{0}\right)$ as

$$
\Gamma \bar{n}\left(\omega_{0}, t_{0}\right)=D\left(t_{0}\right)-\Gamma \bar{n}\left(\omega_{0}\right)
$$

we identify a time-dependent diffusion coefficient $D(t)$ in the last equation (24) as

$$
D(t)=\Gamma \bar{n}\left(\omega_{0}\right)+\left[D\left(t_{0}\right)-\Gamma \bar{n}\left(\omega_{0}\right)\right] e^{-2 \gamma\left(t-t_{0}\right)} .
$$

We thus obtain

$$
\left\langle\tilde{\xi}^{\dagger}(t) \tilde{\xi}\left(t^{\prime}\right)\right\rangle_{N R}=\Gamma \bar{n}\left(\omega_{0}\right)\left[1+r e^{-2 \gamma\left(t-t_{0}\right)}\right] \delta\left(t-t^{\prime}\right)
$$

where we denote

$$
\left.\begin{array}{rl}
\Gamma \bar{n}\left(\omega_{0}\right) & =D(\infty) \\
r & =\frac{D\left(t_{0}\right)}{D(\infty)}-1 \\
& =\frac{\bar{n}\left(\omega_{0}, t_{0}\right)}{\bar{n}\left(\omega_{0}\right)}
\end{array}\right\}
$$


Eq.(26) is the desired nonequilibrium quantum generalization of the fluctuation-dissipation relationship. The classical version of the above equation is given in [6]. This relates instantaneous fluctuations of the nonequilibrium bath (which itself is undergoing relaxation at a rate $\gamma$ due to its coupling with the thermal bath ) to the dissipation of the energy of the system mode through $\Gamma$. The nonequilibrium nature of the bath is implicit in the initial preparation which creates an initial diffusion coefficient $D\left(t_{0}\right)$ and also in the exponentially decaying term.

To check the consistency of the treatment and to allow ourselves a fair comparison with the classical treatment we now make the following comments.

(i) In the steady state limit one recovers the usual fluctuation-dissipation relation for a thermal bath at equilibrium.

(ii) Eq.(26) can also be expressed in terms of energy density fluctuations of the nonequilibrium modes. The energy density which is proportional to the power spectrum centered around $\omega_{0}$ is given by $[\hbar=1]$

$$
\begin{aligned}
u(\Omega, t) & =\frac{\Omega}{4 \pi} \int_{-\infty}^{+\infty} d \tau\left\langle\tilde{\xi}^{\dagger}(t) \tilde{\xi}(t+\tau)\right\rangle e^{i\left(\Omega-\omega_{0}\right) \tau} \\
& =\frac{1}{2} \Omega \bar{n}(\Omega)+e^{-2 \gamma\left(t-t_{0}\right)}\left[u\left(\Omega, t_{0}\right)-\frac{1}{2} \Omega \bar{n}(\Omega)\right] .
\end{aligned}
$$

It is important to note that $t$ is the slow time variable which is well separated from the time-scale of thermal noise. The fluctuations of the noise operator $\xi(t)$ is now explicitly determined by the nonequilibrium state of the bath modes $\left\{c_{\mu}\right\}$ through its energy density $u(\Omega, t)$ at each instant of time. In other words the instantaneous nonequilibrium energy density distribution of fluctuating modes is related to the friction coefficient of these modes on the system degree of freedom through a dynamic equilibrium. The classical version of the above equation can be recovered in the high temperature limit $\left(\bar{n}(\Omega)=1 /[\exp (\Omega / K T)-1] \simeq \frac{K T}{\Omega}\right)$ to obtain

$$
u(\Omega, t)=\frac{1}{2} K T+e^{-2 \gamma\left(t-t_{0}\right)}\left[u\left(\Omega, t_{0}\right)-\frac{1}{2} K T\right] .
$$


This classical version was discussed earlier [6] in the context of classical kinetics of complex systems. Our quantum generalization is more relevant to quantum optical situations as discussed in the next section.

\section{MODIFIED BLOCH EQUATIONS AND TRANSIENT RESONANCE FLUORESCENCE}

We have discussed above a simple solvable model for a nonstationary quantum Markov process and an appropriate generalization of the fluctuation-dissipation relation pertaining to this process. Two immediate consequences are evident. The first one concerns the modification of decay of the Bloch vector components in presence of relaxation of the intermediate bath modes. We show here that the decay is nonexponential in nature so long as the nonstationarity persists following the sudden excitation. The second one centers around the explicit time-dependence of diffusion coefficient due to nonstationarity implied in the fluctuation-dissipation relations (26). The transient noise spectrum of the two-level system is therefore expected to bear this signature of time dependence. With this end in view we calculate the physical spectrum of the two-level system in contact with the nonequilibrium bath driven by a near-resonant classical monochromatic light field. The Hamiltonian of the coupled atom-field system reads as follows;

$$
H=H_{0}+\hbar\left[V \sigma_{+} e^{-i \omega_{c}\left(t-t_{0}\right)}+V \sigma_{-} e^{i \omega_{c}\left(t-t_{0}\right)}\right]
$$

where $H_{0}$ is given by Eq. (1) and $V$ represents the amplitude of the classical pump field with frequency $\omega_{c}$. Proceeding as before, we obtain the Langevin equations for the slowly varying system operators as; 


$$
\left.\begin{array}{lll}
\dot{S}_{+}(t) & = & -(\Gamma-i \delta) S_{+}+2 \xi^{\dagger}(t) S_{z}-2 i V S_{z} \\
\dot{S}_{z}(t) & = & -2 \Gamma\left(S_{z}+\frac{1}{2}\right)-i V S_{+}+i V S_{-}+\xi(t) S_{+}+\xi^{\dagger}(t) S_{-} \\
\dot{S}_{-}(t)= & -(\Gamma+i \delta) S_{-}+2 \xi(t) S_{z}+2 i V S_{z}
\end{array}\right\},
$$

where, $\delta\left(=\omega_{0}-\omega_{c}\right)$ is the detuning and

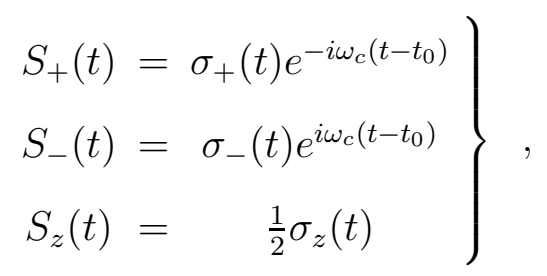

$\xi(t)$ is the noise operator as given by

$$
\xi(t)=i \sum_{\mu} g_{\mu}\left[c_{\mu}^{s}(t)+c_{\mu}\left(t_{0}\right) e^{\left(-i \Omega_{\mu}-\gamma\right)\left(t-t_{0}\right)}\right] e^{i \omega_{c}\left(t-t_{0}\right)}
$$

The noise is characterized by

$$
\begin{aligned}
\langle\xi(t)\rangle_{N R} & =0 \\
\left\langle\xi^{\dagger}(t) \xi\left(t^{\prime}\right)\right\rangle_{N R} & =\Gamma \bar{n}(\nu)\left[1+r e^{-2 \gamma\left(t-t_{0}\right)}\right] \delta\left(t-t^{\prime}\right) .
\end{aligned}
$$

While considering the above equations we emphasize again the separation of time scales $\gamma \ll \Gamma$.

As a next step, we construct the following Bloch equations for one-time averages from the Langevin equations (31) [ this requires the calculation of averages like $\left\langle\xi^{\dagger}(t) S_{z}\right\rangle$ which include nonstationary contribution involving Eq.(34) as shown in Appendix-A ],

$$
\begin{aligned}
\left\langle\dot{S}_{+}(t)\right\rangle_{N R}= & -(\Gamma-i \delta)\left\langle S_{+}(t)\right\rangle_{N R}+2 \Gamma \bar{n} r e^{-2 \gamma t}\left\langle S_{+}(t)\right\rangle_{N R}-2 i V\left\langle S_{z}(t)\right\rangle_{N R}, \\
\left\langle\dot{S}_{-}(t)\right\rangle_{N R}= & -(\Gamma+i \delta)\left\langle S_{-}(t)\right\rangle_{N R}+2 \Gamma(\bar{n}+1) r e^{-2 \gamma t}\left\langle S_{-}(t)\right\rangle_{N R}+2 i V\left\langle S_{z}(t)\right\rangle_{N R}, \\
\left\langle\dot{S}_{z}(t)\right\rangle_{N R}= & -2 \Gamma\left[\left\{1-(2 \bar{n}+1) r e^{-2 \gamma t}\right\}\left\langle S_{z}(t)\right\rangle_{N R}+\frac{1}{2}\right] \\
& -i V\left\langle S_{+}(t)\right\rangle_{N R}+i V\left\langle S_{-}(t)\right\rangle_{N R} .
\end{aligned}
$$


The following comments should be made about the Eqs.(35-37) supplemented by Eq.(34) :

(i) The exponential term in Eq.(34) results in an effective transient modification of decay rates of all the Bloch vector components. In the long time limit one, however, recovers the usual decay rates and the standard Bloch equations. It is also interesting to note that the two polarization components $\left\langle S_{+}\right\rangle$and $\left\langle S_{-}\right\rangle$decay at different rates in contrast to the usual case of equilibrium bath.

(ii) The nonstationary contributions in the Bloch equations immediately assert that in absence of the driving fields $(V=0)$ the decay of the polarization components is non-exponential in nature. This is reminiscent of what has been observed in the relaxation kinetics of classical complex systems where the influence of an initial nonequilibrium excitation of others degrees of freedom of a complex system on the relaxation of a specific quantity of interest has been explored.

(iii) Although the noise correlation in Eq.(34) involves an exponentially decaying term, $\delta\left(t-t^{\prime}\right)$ makes the noise instantaneously correlated. This implies that we consider here a broad-band reservoir instead of a colored bath. It is important to note that Lewenstein et.al. [8] in a different context have considered earlier the atomic decay in presence of a colored reservoir. They have used the modified Bloch equations in non-Markovian form (which involves exponentially decaying terms due to the finite response time of the reservoir) and shown how the effects of the colored reservoir can be inhibited at large driving fields. Thus the origin of the exponential term in Eq.(34) is different.

We now turn to the second issue, i.e., the calculation of the transient resonance fluorescent spectra. Using matrix notation, the above three equations (35-37) for single time expectation values can be put in a compact form as;

$$
\frac{d \mathbf{u}(t)}{d t}=\mathbf{M}(\mathbf{t}) \mathbf{u}(t)+\mathbf{f}
$$

where $\mathbf{u}(t)$ and $\mathbf{f}$ are the column vectors and are given by 


$$
\mathbf{u}=\left(\begin{array}{c}
\left\langle S_{+}(t)\right\rangle_{N R} \\
\left\langle S_{z}(t)\right\rangle_{N R} \\
\left\langle S_{-}(t)\right\rangle_{N R}
\end{array}\right) \quad \text { and } \quad \mathbf{f}=\left(\begin{array}{c}
0 \\
-\Gamma \\
0
\end{array}\right)
$$

with

$$
\mathbf{M}(\mathbf{t})=\left(\begin{array}{ccc}
-(\Gamma-i \delta)+2 \Gamma \bar{n} r e^{-2 \gamma t} & -2 i V & 0 \\
-i V & -2 \Gamma+2 \Gamma r(2 \bar{n}+1) e^{-2 \gamma t} & i V \\
0 & 2 i V & -(\Gamma+i \delta)+2 \Gamma(\bar{n}+1) r e^{-2 \gamma t}
\end{array}\right) .
$$

Since the calculation of spectra rests on the evaluation of two-time correlation functions of the atomic operators it is essential to examine the validity of quantum regression hypothesis in the present context. To this end we note the following points.

The essential statistical properties of the intermediate bath modes are contained in Eq.(34). This equation suggests a differential behavior in time dependence of the two terms. First, the exponential time dependence is due to the initial preparation at $t_{0}$ and subsequent relaxation at any time $\left(t-t_{0}\right.$ ) of the intermediate bath modes. So the nonstationary nature is implied in this term. On the other hand the $\delta\left(t-t^{\prime}\right)$ term essentially signifies the correlation of intermediate bath fluctuations, $\xi(t)$, at times $t$ and $t^{\prime}$. The presence of $\delta\left(t-t^{\prime}\right)$ ensures the broad band nature and hence the Markov property of the bath. These statistical considerations, therefore, reveal that the dynamics of the two-level atom is acted upon by a nonstationary but Markovian stochastic process due to the intermediate oscillators. By using the Langevin description of Heisenberg equation of motion, Lax [9] has proved that Markov property implies regression theorem as well the converse. The Markov property is defined by the requirement that a Langevin force at time $t$ is uncorrelated to any information at earlier time $t^{\prime}$. The regression hypothesis is a consequence of this requirement. Lax has specifically shown [9] that Onsager's original statement for an equilibrium situation is valid even for a nonequilibrium situation provided the system is Markovian. The validity of regression hypothesis therefore implies that two- 
time correlation evolves in the same way as one-time expectation value.

The equation for evolution of two-time correlation functions is then given by

$$
\frac{d}{d \tau} \mathbf{v}\left(t_{2}, \tau\right)=\mathbf{M}\left(t_{2}, \tau\right) \mathbf{v}\left(t_{2}, \tau\right)+\mathbf{F}\left(t_{2}\right),
$$

where

$$
\mathbf{v}\left(t_{2}, \tau\right)=\left(\begin{array}{c}
\left\langle S_{+}\left(t_{2}+\tau\right) S_{-}\left(t_{2}\right)\right\rangle_{N R} \\
\left\langle S_{z}\left(t_{2}+\tau\right) S_{-}\left(t_{2}\right)\right\rangle_{N R} \\
\left\langle S_{-}\left(t_{2}+\tau\right) S_{-}\left(t_{2}\right)\right\rangle_{N R}
\end{array}\right), \mathbf{F}\left(t_{2}\right)=\left(\begin{array}{c}
0 \\
-\Gamma\left\langle S_{-}\left(t_{2}\right)\right\rangle_{N R} \\
0
\end{array}\right)
$$

The relevant correlation function $v_{1}\left(t_{2}, \tau\right)$ required for calculation of spectra is the first component of the vector $\mathbf{v}\left(t_{2}, \tau\right)$ and is given by

$$
v_{1}\left(t_{2}, \tau\right)=\left\langle S_{+}\left(t_{2}, \tau\right) S_{-}\left(t_{2}\right)\right\rangle_{N R} .
$$

We assume that the atom is initially in its ground state. The Eq.(41) is then solved to calculate the correlation function (43). The details are given in the Appendix-B.

At this point it must be emphasized that since we are dealing with a nonstationary situation the standard steady state definition of spectrum is not adequate to describe the transient spectral features. We therefore resort to a non-steady state spectrum or the so called 'physical spectrum' of the emission from the two-level atoms, where the attention is focused on a dynamic evolution of the spectrum following an abrupt excitation of the atom and the intermediate oscillator modes. The main reason for studying the time-dependent spectrum is that the familiar power spectrum which results from Weiner-Khintchine theorem is not applicable to nonstationary processes. Eberly and Wodkiewicz [10] have shown that the suitably normalized counting rate of a photo detector can be used to define a time-dependent spectrum. This definition allows the influence of the spectrum analyzer ( basically a Fabry-Perot interferometer, for example ) to be exhibited in the spectrum so that the band limit of the measuring device is appropriately incorporated which makes the spectrum 
free from ambiguities. It has also been emphasized [10] that when the instrumental width, $W$, is narrow enough such that $W \ll \Gamma$ the spectrum appears to be qualitatively close to Weiner-Khintchine spectrum. This transient spectrum has been used previously on several occasions [11]. Following Eberly and Wodkiewicz [10] we define the time-dependent spectrum in terms of the time correlation function $v_{1}\left(t_{2}, \tau\right)$ as follows;

$$
S(t, \omega, W)=2 W R e \int_{0}^{t} d t_{2} e^{-W\left(t-t_{2}\right)} \int_{0}^{t-t_{2}} d \tau e^{\left(\frac{W}{2}-i \Delta\right) \tau} v_{1}\left(t_{2}, \tau\right)
$$

Here $t$ is the elapsed time after the system and the intermediate oscillator modes have been subjected to the initial excitation at $t=t_{0}(=0), W$ is the full width of the transmission peak of the interferometer and $\Delta\left(=\omega-\omega_{c}\right)$ is the detuning, or frequency offset of the Fabry-Perot line center above the frequency of the field $\omega_{c}$. It is important to note that the time-dependent spectrum is expressed in terms of the two integrals. The first integral is over the correlation time $\tau$ and is actually the counterpart of Weiner-Khintchine spectrum band limited by the width $W$ of the measuring device, while the second one over $t_{2}$ takes into account of the nonstationarity which makes the spectrum $t$-dependent.

Making use of Eq.(43) in Eq.(44), performing the integration over $\tau$ and $t_{2}$ and extracting the real part, we obtain numerically the time-dependent spectrum as discussed below.

Since the excitation at $t=0$, prepares an initial nonequilibrium energy density of the intermediate oscillator modes which differs from its equilibrium value, the initial diffusion coefficient $D(0)$ deviates from its stationary long time value $D(\infty)$. This deviation is measured in terms of $r\left(=\frac{D(0)}{D(\infty)}-1\right)$ [see Eq.(27)] or equivalently in terms of the ratio of the photon numbers $\frac{\bar{n}\left(\omega_{0}, t_{0}\right)}{\bar{n}\left(\omega_{0}\right)}$. Another quantity of interest is the rate of relaxation $\gamma$ of the nonequilibrium intermediate oscillator modes due to their coupling to the thermal modes. Both $r$ and $\gamma$ contribute significantly to the nonequilibrium version of fluctuation-dissipation relation [ Eq.(26)] which is essential for understanding the influence of a nonequilibrium bath on the transient fluorescence spectrum. In Fig.(1) we plot the physical spectra 
at three different times after the initial excitation at $t=0$ for the parameter set $r=0.4, \bar{n}=0.1$, $\Gamma=1.0, \gamma=0.1$ under resonance condition $\delta=0$ and instrumental linewidth $W=4.0$ for a low value of field strength $V=2.5$. The choice of parameter space is guided by the early work of Eberly and Wodkiewicz [10] on the physical spectra of resonance fluorescence. For a higher value of $V(=10.0)$ the expected three-peak Mollow structure appears as shown in Fig.(2) ( all other parameters kept fixed ). The steady state is clearly achieved around $t=10.0$. It is interesting to note that in the spectra showed in Figs.(1) and (2), the asymptotic condition corresponds to the usual stationary bath case, presenting the narrowest line. The transient nature of broadening of the bands is due to the creation of a nonequilibrium bath mode energy density through $r$. Since $r$ is a measure of the excess energy gained by the intermediate modes into which the system has to release its energy, the system also draws some energy from these modes by virtue of the fluctuation-dissipation relation. The latter relation illustrates a dynamical balance of inward flow of energy due to fluctuations from the reservoir into the system and the outward flow of energy from the system to the reservoir due to dissipation of the system mode. This nonstationary diffusion of fluctuations from the intermediate bath modes into the system leads to transient spectral broadening. This persists so long as the nonstationarity

remains. If the relaxation of these bath modes approaches the time scale of $\frac{1}{\Gamma}$ ( with increase of $\gamma$ ) the broadening effect no longer appears. In the next section we describe an experimental scheme to show how this can be realized within the purview of a simple cavity QED experiment.

\section{DISCUSSION OF AN EXPERIMENTAL SCHEME AND CONCLUSIONS}

Based on a microscopic model for a nonequilibrium bath we have constructed the modified Bloch equations which incorporate the effect of nonstationary relaxation and calculated the transient resonance fluorescence spectra of a two-level system driven by a near resonant strong classical field. 
We now discuss a specific system presenting the transient broadening effect studied in this paper. It is wellknown that the spectrum of the radiation emitted by a strongly driven system is considerably modified if the atoms are confined in a cavity. For our purpose the dynamics may be conveniently described if one considers a two-level Rydberg atom as a system contained in a cavity ( whose modes serve as the intermediate oscillator modes of the present model ). The cavity in turn is weakly coupled to the vacuum modes playing the role of the equilibrium reservoir through the cavity losses. By sudden sweeping of the resonance of the cavity it is possible to dump an appreciable amount of energy on the cavity modes by changing the number of photons abruptly. ( The tuning of cavity in studying the emission of strongly driven two-level systems like Ba atoms into the modes of the cavity had been experimentally carried out both under adiabatic [12] and non-adiabatic [13, 14] conditions in cavity QED experiments [11 13]. ) This corresponds to the initial preparation of the nonstationary state of the cavity modes by changing $r\left[=\frac{\bar{n}\left(\omega_{0}, t_{0}\right)}{\bar{n}\left(\omega_{0}\right)}\right.$, see Eqs.(26) and (27)] in such a way that the energy of these modes becomes suddenly higher than the average energy. Once this nonstationarity is attained, the effect of relaxation of the cavity modes on the emission of the strongly driven ( externally ) two-level atoms can be monitored by observing the transient fluorescence spectrum. Since the atom-cavity interaction ( $\Gamma$, say $\sim 100 \mathrm{MHz}$ ) is strong compared to the decay rate of the cavity modes ( $\gamma$, say $\sim 20 \mathrm{MHz}$ ), the separation of time scales as required can be conveniently maintained. We also expect to observe the nonexponential decay of emission of the excited two-level atom into the modes of an optical cavity so long as the nonstationarity persists.

We now summarize the main conclusions of this study:

(i) The microscopic model proposed here may serve as a simple solvable paradigm for a nonstationary quantum Markov process.

(ii) We establish an appropriate generalization of the fluctuation-dissipation relation and its classical correspondence pertaining to the above-mentioned process.

(iii) The origin of nonstationarity (or nonequilibrium nature of the bath) lies in the creation and 
subsequent relaxation of an energy density fluctuation distribution function of the intermediate bath modes following a sudden excitation.

(iv) Keeping in view of the systematic separation of time-scales involved in the overall dynamics we have shown that the decay of the polarization and population inversion components of the Bloch vector is non-exponential so long as the nonstationarity persists.

(v) The nonstationarity of the bath results in time dependence of the diffusion coefficient which show up in the transient broadening of the physical spectra of resonance fluorescence.

(vi) We have outlined a simple experimental scheme within a cavity QED setup to verify the aspects nonexponential decay and transient broadening of emissions from a strongly driven two-level system in a cavity.

Since the underlying model of relaxation employed here bears its origin in complex coupled systems one may also envisage guest-host systems embedded in a lattice ( where the immediate local neighborhood of the guest comprises intermediate oscillator modes and the lattice plays the role of a thermal bath ) as typical candidates for experimental realization of such transient fluorescent processes. We thus expect the model to be relevant in the context of single molecule spectroscopy [15.

\section{ACKNOWLEDGMENTS}

Partial financial support by the Department of Science and Technology, Govt. of India, is thankfully acknowledged. DSR is indebted to Professor G. S. Agarwal for discussions. 


\section{APPENDIX A: CALCULATION OF THE AVERAGE $\left\langle\xi^{\dagger}(T) S_{Z}(T)\right\rangle_{N R}$}

To calculate $\left\langle\xi^{\dagger}(t) S_{z}(t)\right\rangle_{N R}$ we proceed as follows :

We have from Eq.(36)

$$
\xi^{\dagger}(t)=-i \sum_{\mu} g_{\mu}\left[c_{\mu}^{s \dagger}(t)+c_{\mu}^{\dagger}\left(t_{0}\right) e^{\left(i \Omega_{\mu}-\gamma\right)\left(t-t_{0}\right)}\right] e^{-i \omega_{c}\left(t-t_{0}\right)}
$$

This can be written as

$$
\xi^{\dagger}(t)=\xi^{s \dagger}(t)+\xi^{\dagger N}(t)
$$

where, $\xi^{s \dagger}(t)=-i \sum_{\mu} g_{\mu} c_{\mu}^{s \dagger}(t) e^{-i \omega_{c}\left(t-t_{0}\right)}$ represents the stationary ( long time ) fluctuation and

$$
\xi^{\dagger N}(t)=-i \sum_{\mu} g_{\mu} c_{\mu}^{\dagger}\left(t_{0}\right) e^{\left(i \Omega_{\mu}-\gamma\right)\left(t-t_{0}\right)} e^{-i \omega_{c}\left(t-t_{0}\right)}
$$

denotes the fluctuations due to the coupling of the system with the relaxing modes. It is essential to note that because of the relaxation $\xi^{\dagger N}(t)$ noise is nonstationary. It is important to note that the separation of time scales of $\xi^{s^{\dagger}}(t)$ and $\xi^{\dagger N}(t) . \xi^{s^{\dagger}}(t)$ is much faster compared to $\xi^{\dagger N}(t)$ and represents a Gaussian white noise. Also $\xi^{s^{\dagger}}(t)$ and $\xi^{\dagger N}(t)$ are assumed uncorrelated [6].

Thus we note

$$
\left\langle\xi^{\dagger}(t) S_{z}(t)\right\rangle_{N R}=\left\langle\xi^{s \dagger}(t) S_{z}(t)\right\rangle_{N R}+\left\langle\xi^{\dagger N}(t) S_{z}(t)\right\rangle_{N R}
$$

Since, $\xi^{s \dagger}(t)$ is much faster and describes a stationary process, we write

$$
\left\langle\xi^{s \dagger}(t) S_{z}(t)\right\rangle_{N R}=\left\langle\xi^{s \dagger}(t)\right\rangle_{N R}\left\langle S_{z}(t)\right\rangle_{N R}=0
$$

Thus we have

$$
\left\langle\xi^{\dagger}(t) S_{z}(t)\right\rangle_{N R}=\left\langle\xi^{\dagger N}(t) S_{z}(t)\right\rangle_{N R}
$$


Because of the exponential term $\exp \left[-\gamma\left(t-t_{0}\right)\right]$ in the expression of $\xi^{\dagger N}(t)$, expression (A2) describes a nonstationary average which cannot be equated to zero as shown below.

Making use of the identity

$$
S_{z}(t)=S_{z}(t-\Delta t)+\int_{t-\Delta t}^{t} d t^{\prime} \dot{S}_{z}\left(t^{\prime}\right)
$$

and the expression for $\dot{S}_{z}(t)$, we get

$$
\begin{aligned}
S_{z}(t) & =S_{z}(t-\Delta t) \\
& +\int_{t-\Delta t}^{t} d t^{\prime}\left[-2 \Gamma\left\{S_{z}\left(t^{\prime}\right)+\frac{1}{2}\right\}-i V S_{+}\left(t^{\prime}\right)+i V S_{-}\left(t^{\prime}\right)+\xi\left(t^{\prime}\right) S_{+}\left(t^{\prime}\right)+\xi^{\dagger}\left(t^{\prime}\right) S_{-}\left(t^{\prime}\right)\right] \\
& =S_{z}(t-\Delta t) \\
& +\int_{t-\Delta t}^{t} d t^{\prime}\left[-2 \Gamma\left\{S_{z}\left(t^{\prime}\right)+\frac{1}{2}\right\}-i V S_{+}\left(t^{\prime}\right)+i V S_{-}\left(t^{\prime}\right)+\xi^{s}\left(t^{\prime}\right) S_{+}\left(t^{\prime}\right)+\xi^{N}\left(t^{\prime}\right) S_{+}\left(t^{\prime}\right)\right. \\
& \left.+\xi^{s \dagger}\left(t^{\prime}\right) S_{-}\left(t^{\prime}\right)+\xi^{N \dagger}\left(t^{\prime}\right) S_{-}\left(t^{\prime}\right)\right] .
\end{aligned}
$$

We then calculate the average

$$
\left\langle\xi^{N \dagger}(t) S_{z}(t)\right\rangle_{N R}=\int_{t-\Delta t}^{t} d t^{\prime}\left\langle\xi^{N \dagger}(t) \xi^{N}\left(t^{\prime}\right) S_{+}\left(t^{\prime}\right)\right\rangle_{N R}
$$

where we make use of the fact that $\xi^{s^{\dagger}}(t)$ and $\xi^{\dagger N}(t)$ are uncorrelated [6] and the operator $S_{z}\left(t^{\prime}\right)$ at time $t^{\prime}$ is not affected by fluctuation at a latter time $t$. Following Bourret [16]17] and van Kampen 18] we now make decoupling approximation ( which implies that the correlation of fluctuations $\xi^{N}(t)$ is much short compared to the coarsed-grained time scale over which the average $\left\langle S_{+}\right\rangle$evolves in time ) to obtain

$$
\left\langle\xi^{N \dagger}(t) \xi^{N}\left(t^{\prime}\right) S_{+}\left(t^{\prime}\right)\right\rangle_{N R}=\left\langle\xi^{N \dagger}(t) \xi^{N}\left(t^{\prime}\right)\right\rangle_{N R}\left\langle S_{+}\left(t^{\prime}\right)\right\rangle_{N R}
$$

We then use the fluctuation-dissipation relation for the $\xi^{N}(t)$ [ Eq.(37) ] to obtain

$$
\left\langle\xi^{N \dagger}(t) S_{z}(t)\right\rangle_{N R}=\Gamma \bar{n} r e^{-2 \gamma t}\left\langle S_{+}(t)\right\rangle_{N R}
$$


We thus take note in passing that identification of a nonstationary part $\xi^{\dagger N}(t)$ (which is not invariant under time translation) in Eq.(A1) leads us to a non zero average like (A3). The other nonstationary averages are similarly calculated.

\section{APPENDIX B: CALCULATION OF TWO-TIME CORRELATION FUNCTIONS}

The matrix $\mathbf{M}$ as defined in Eq.(40) may be rewritten as

$$
\mathbf{M}(\mathbf{t})=\mathbf{M}_{\mathbf{0}}+2 \Gamma r e^{-2 \gamma t} \mathbf{M}_{\mathbf{1}}
$$

where

$$
\begin{aligned}
& \mathbf{M}_{\mathbf{0}}=\left(\begin{array}{ccc}
-(\Gamma-i \delta) & -2 i V & 0 \\
-i V & -2 \Gamma & i V \\
0 & 2 i V & -(\Gamma+i \delta)
\end{array}\right), \\
& \mathbf{M}_{\mathbf{1}}=\left(\begin{array}{ccc}
\bar{n} & 0 & 0 \\
0 & 2 \bar{n}+1 & 0 \\
0 & 0 & \bar{n}+1
\end{array}\right) .
\end{aligned}
$$

The solution of Eq.(41) in terms of $\mathbf{M}_{\mathbf{0}}$ and $\mathbf{M}_{\mathbf{1}}$ is

$$
\begin{aligned}
\mathbf{v}\left(t_{2}, \tau\right)= & \exp \left[\mathbf{M}_{0} \tau-\frac{\Gamma}{\gamma} r e^{-2 \gamma\left(t_{2}+\tau\right)} \mathbf{M}_{1}\right] \\
& \times\left\{\mathbf{v}\left(t_{2}, 0\right)+\int_{0}^{\tau} d t \exp \left[-\mathbf{M}_{0} t+\frac{\Gamma}{\gamma} r e^{-2 \gamma\left(t_{2}+\tau\right)} \mathbf{M}_{1}\right]\right\} \mathbf{F}\left(t_{2}\right) .
\end{aligned}
$$

We assume that the atom is initially in its ground state. Then,

$$
\mathbf{v}\left(t_{2}, 0\right)=\left(\begin{array}{c}
\frac{1}{2}\left(1+2\left\langle S_{z}\right\rangle_{N R}\right) \\
-\frac{1}{2}\left\langle S_{-}\right\rangle_{N R} \\
0
\end{array}\right), \mathbf{u}(0)=\left(\begin{array}{c}
0 \\
-\frac{1}{2} \\
0
\end{array}\right)
$$


where $\mathbf{v}\left(t_{2}, \tau\right)$ and $\mathbf{u}(t)$ are defined by Eqs.(42) and (39) respectively.

Defining a matrix $\mathbf{T}$ and a vector $\mathbf{g}$ as

$$
\mathbf{T}=\left(\begin{array}{ccc}
0 & 1 & 0 \\
0 & 0 & -\frac{1}{2} \\
0 & 0 & 0
\end{array}\right), \mathbf{g}=\left(\begin{array}{c}
\frac{1}{2} \\
0 \\
0
\end{array}\right)
$$

we can write

$$
\mathbf{v}\left(t_{2}, 0\right)=\mathbf{T} \mathbf{u}\left(t_{2}\right)+\mathbf{g}
$$

Hence from Eq.(B4), using Eq.(B5) we get

$$
\begin{aligned}
\mathbf{v}\left(t_{2}, \tau\right)= & \exp \left[\mathbf{M}_{0} \tau-\frac{\Gamma}{\gamma} r e^{-2 \gamma\left(t_{2}+\tau\right)} \mathbf{M}_{1}\right] \\
& \times\left\{\left[\mathbf{T u}\left(t_{2}\right)+\mathbf{g}\right]+\int_{0}^{\tau} d t \exp \left[-\mathbf{M}_{0} t+\frac{\Gamma}{\gamma} r e^{-2 \gamma\left(t_{2}+\tau\right)} \mathbf{M}_{1}\right]\right\} \mathbf{F}\left(t_{2}\right) .
\end{aligned}
$$

The solution of Eq.(38) is

$$
\begin{aligned}
\mathbf{u}\left(t_{2}\right)= & \exp \left[\mathbf{M}_{0} t_{2}-\frac{\Gamma}{\gamma} r e^{-2 \gamma t_{2}} \mathbf{M}_{1}\right] \\
& \times\left\{\mathbf{u}(0)+\int_{0}^{t_{2}} d t \exp \left[-\mathbf{M}_{0} t+\frac{\Gamma}{\gamma} r e^{-2 \gamma t} \mathbf{M}_{1}\right]\right\} \mathbf{f} .
\end{aligned}
$$

We again define a matrix

$$
\mathbf{K}=\left(\begin{array}{ccc}
0 & 0 & 0 \\
0 & 0 & -\Gamma \\
0 & 0 & 0
\end{array}\right)
$$

to write 


$$
\mathbf{F}\left(t_{2}\right)=\mathbf{K} \mathbf{u}\left(t_{2}\right)
$$

Using Eq.(B7) and Eq.(B8) we get from Eq.(B6), the solution for two-time correlation function in terms of the initial condition as follows;

$$
\begin{aligned}
\mathbf{v}\left(t_{2}, \tau\right)= & \exp \left(\mathbf{M}_{0} \tau-\frac{\Gamma}{\gamma} r e^{-2 \gamma\left(t_{2}+\tau\right)} \mathbf{M}_{1}\right)\left[\mathbf { T } \left[\left\{\exp \left(\mathbf{M}_{0} t_{2}-\frac{\Gamma}{\gamma} r e^{-2 \gamma t_{2}} \mathbf{M}_{1}\right)\right\} \mathbf{u}(0)\right.\right. \\
& \left.\left.+\exp \left(\mathbf{M}_{0} t_{2}-\frac{\Gamma}{\gamma} r e^{-2 \gamma t_{2}} \mathbf{M}_{1}\right) \int_{0}^{t_{2}} d t \exp \left(-\left\{\mathbf{M}_{0} t-\frac{\Gamma}{\gamma} r e^{-2 \gamma t} \mathbf{M}_{1}\right\}\right) \mathbf{f}\right]+\mathbf{g}\right] \\
& +\exp \left(\mathbf{M}_{0} \tau-\frac{\Gamma}{\gamma} r e^{-2 \gamma\left(t_{2}+\tau\right)} \mathbf{M}_{1}\right)\left\{\int_{0}^{\tau} d t \exp \left(-\left\{\mathbf{M}_{0} t-\frac{\Gamma}{\gamma} r e^{-2 \gamma\left(t_{2}+\tau\right)} \mathbf{M}_{1}\right\}\right)\right\} \\
& \mathbf{K}\left\{\exp \left(\mathbf{M}_{0} t_{2}-\frac{\Gamma}{\gamma} r e^{-2 \gamma t_{2}} \mathbf{M}_{1}\right) \mathbf{u}(0)\right. \\
& \left.+\exp \left(\mathbf{M}_{0} t_{2}-\frac{\Gamma}{\gamma} r e^{-2 \gamma t_{2}} \mathbf{M}_{1}\right) \int_{0}^{t_{2}} d t \exp \left(-\left\{\mathbf{M}_{0} t-\frac{\Gamma}{\gamma} r e^{-2 \gamma t} \mathbf{M}_{1}\right\}\right) \mathbf{f}\right\}
\end{aligned}
$$




\section{REFERENCES}

[1] W. H. Louisell, Quantum Statistical Properties of Radiation (Wiley, New York 1973) ; M. Lax, Phys. Rev. 145, 110 (1966) ; M. Lax and H. Yuen, Phys. Rev. 172, 362 (1968) ; G. S. Agarwal, Phys. Rev. A2, 2038 (1970) ; R. Graham and H. Haken, Z. Phys. 235, 166 (1970).

[2] A. Caldeira and A. J. Leggett, Ann. Phys. 149, 374 (1983).

[3] See for example, a review, G. Gangopadhyay and D. S. Ray, in Advances in Multiphoton Processes and Spectroscopy, edited by S. H. Lin, A. A. Villayes and F. Fujimura (World Scientific, Singapore, 1993) vol.8 ; G. Gangopadhyay and D. S. Ray, Phys.Rev. A46, 1507 (1992); A43, 6424 (1991) ; J. Chem. Phys. 96, 4693 (1992).

[4] N. Lu and J. Bergou, Phys. Rev. A40, 237 (1989) .

[5] C. Gardiner and M. J. Collet, Phys. Rev. A31, 3761 (1985); A. E. Ekert and P. L. Knight, Phys. Rev. A42, 487 (1990); Z. Ficek and B. C. Sanders, J. Phys. B27, 809 (1994) ; A. S. Parkins, Phys. Rev. A42, 4352 (1990).

[6] J. Ray Chaudhuri, G. Gangopadhyay and D. S. Ray, J. Chem. Phys. 109, 5565 (1998) ; M. Millonas and C. Ray, Phys. Rev. Letts. 751110 (1995).

[7] R. Landauer, J. Stat. Phys. 9351 (1973); 11525 (1974); 131 (1975); D. L. Stein, R. Doering, R. G. Palmer, J. L. van Hemmen and R. M. McLaughlin, Phys. Letts. A136, 353 (1989).

[8] M. Lewenstein, T. W. Mossberg and R. J. Glauber, Phys. Rev. Letts. 59, 775 (1987).

[9] M. Lax, Phys. Rev. 157, 213 (1967).

[10] J. H. Eberly and K. Wodkiewicz, J. Opt. Soc. Am. 67, 1252 (1977).

[11] B. Deb and D. S. Ray, Phys. Rev. A49, 5015 (1994); J. H. Eberly, C. V. Kunasz and K. 
Wodkiewicz, J. Phys. B13, 217 (1980); B. Renand, R. M. Whitley and C. R. Stroud, J. Phys. B9, L19 (1977).

[12] D. Holm and M. Sargent III, Phys. Rev. A33, 4001 (1986).

[13] Y. Zhu, A. Lezama and T. W. Mossberg, Phys. Rev. A39, 2268 (1989).

[14] G. S. Agarwal, Phys. Rev. A41, 2886 (1990).

[15] Ph. Tamarat, B. Lounis, J. Bernard, M. Orrit, S. Kummer, R. Kettner, S. Mais and T. Basche, Phys. Rev. Letts. 75, 1514 (1995) ; B. Lounis, F. Jelezko and M. Orrit, Phys. Rev. Letts. 78, 3673 (1997).

[16] R. C. Bourret, Can. J. Phys. 40, 782 (1962).

[17] A. Brissaud and U. Frisch, J. Math. Phys. 15, 524 (1974).

[18] N. G. van Kampen, Phys. Rep. 24, 171 (1976). 


\section{Figure Captions}

1. Fig.1 : Time-dependent resonance fluorescence spectra of the two-level system for different dimensionless times with $\mathrm{W}=4.0, \delta=0.0, \bar{n}=0.1, \mathrm{r}=0.4, \gamma=0.1$ and $\mathrm{V}=2.5$ (scales arbitrary).

2. Fig.2 : Time-dependent resonance fluorescence spectra of the two-level system for different dimensionless times with $\mathrm{W}=4.0, \delta=0.0, \bar{n}=0.1, \mathrm{r}=0.4, \gamma=0.1$ and $\mathrm{V}=10.0$ (scales arbitrary). 


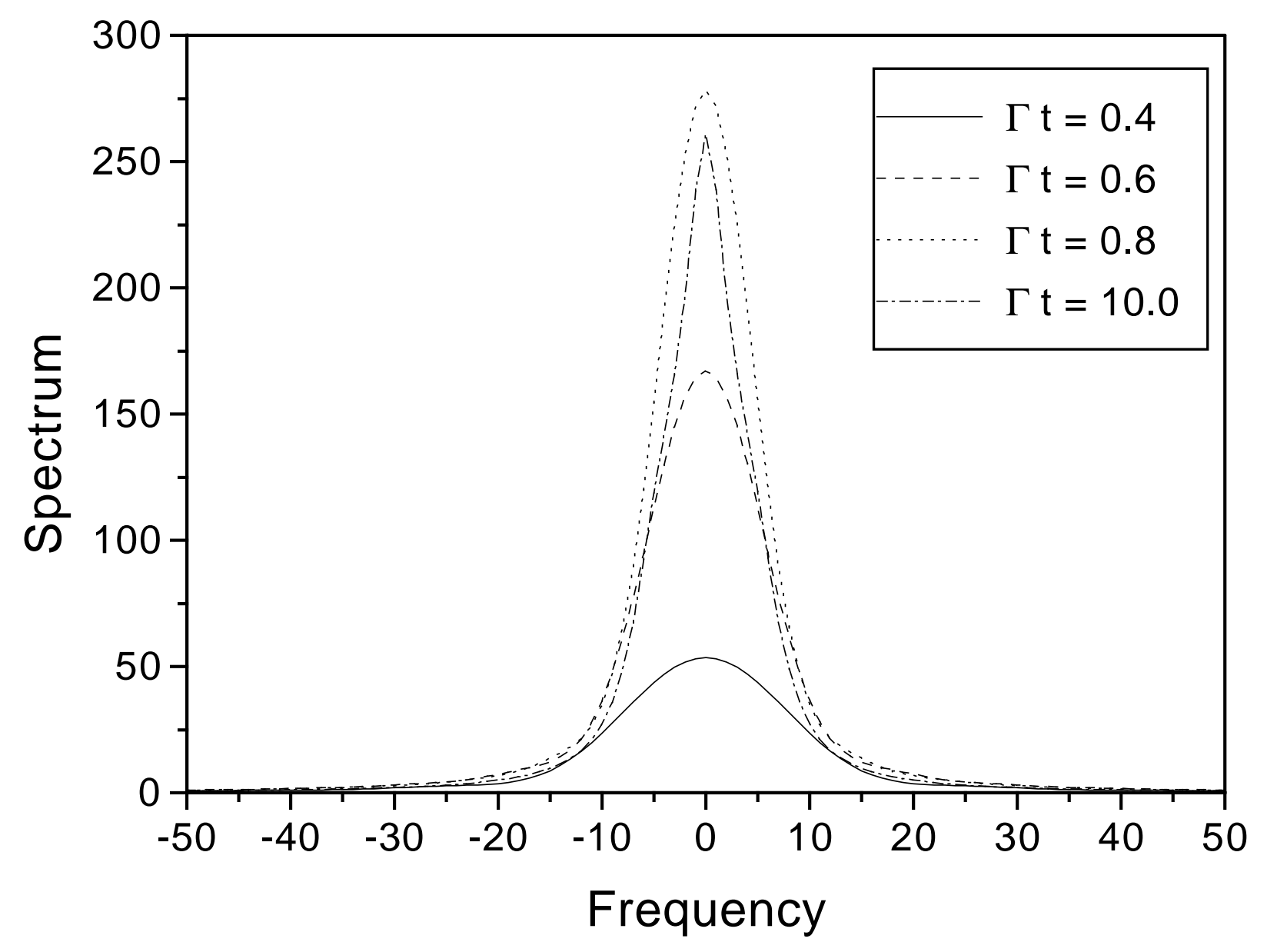

Fig. 1 


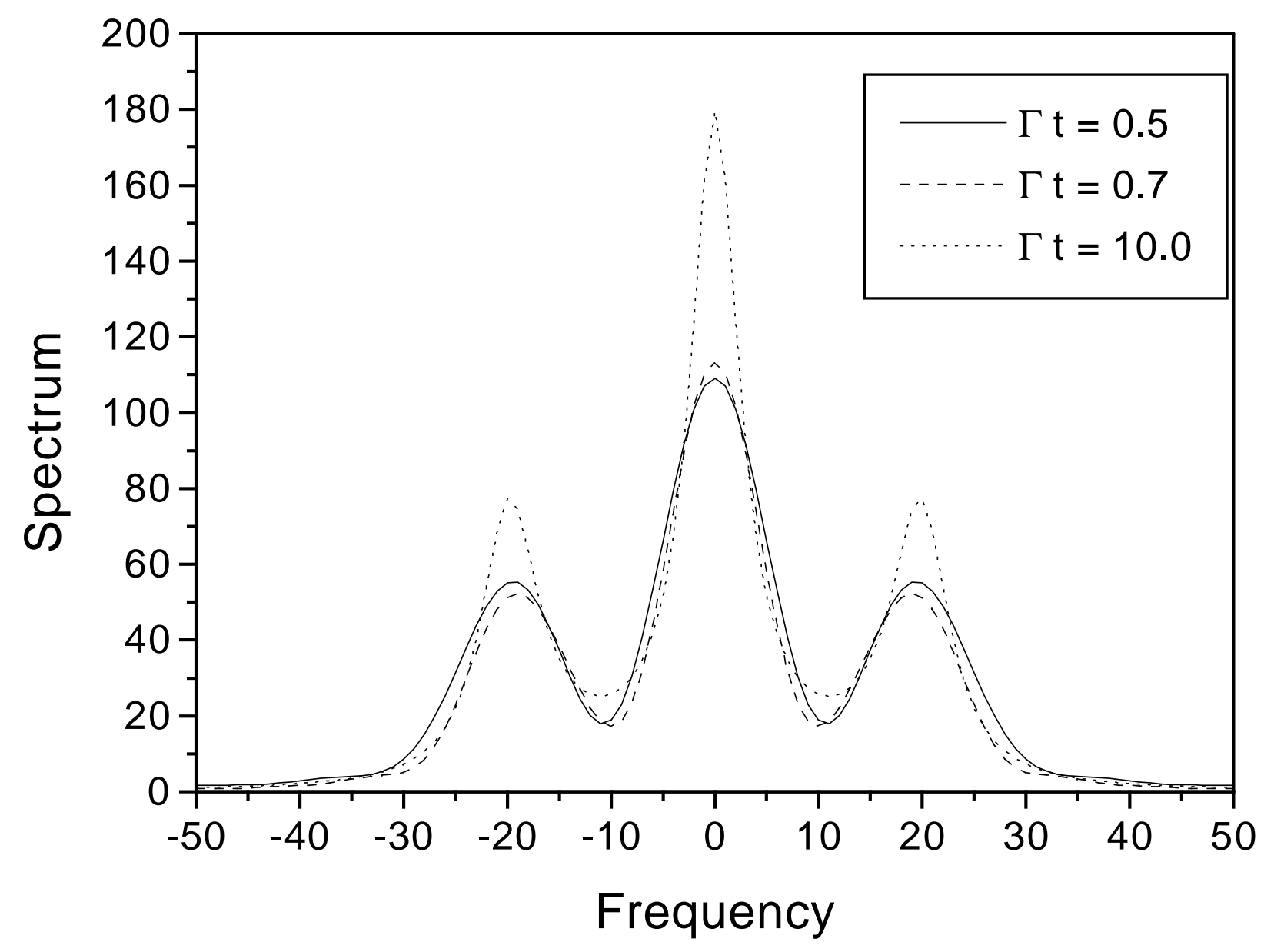

Fig.2 